PROFESIONALES Y HERRAMIENTAS PARA EL DESARROLLO LOCAL Y SUS SINERGIAS TERRITORIALES. EVALUACIÓN Y PROPUESTAS DE FUTURO IX Coloquio Nacional de Desarrollo Local del GTDL-AGE 

ANTONIO MARTÍNEZ PUCHE, XAVIER AMAT MONTESINOS, ISABEL SANCHO CARBONELL y DANIEL SANCHIZ CASTAÑO (EDS.)

\section{PROFESIONALES Y HERRAMIENTAS PARA EL DESARROLLO LOCAL Y SUS SINERGIAS TERRITORIALES. EVALUACIÓN Y PROPUESTAS DE FUTURO}

IX Coloquio Nacional de Desarrollo Local del GTDL-AGE

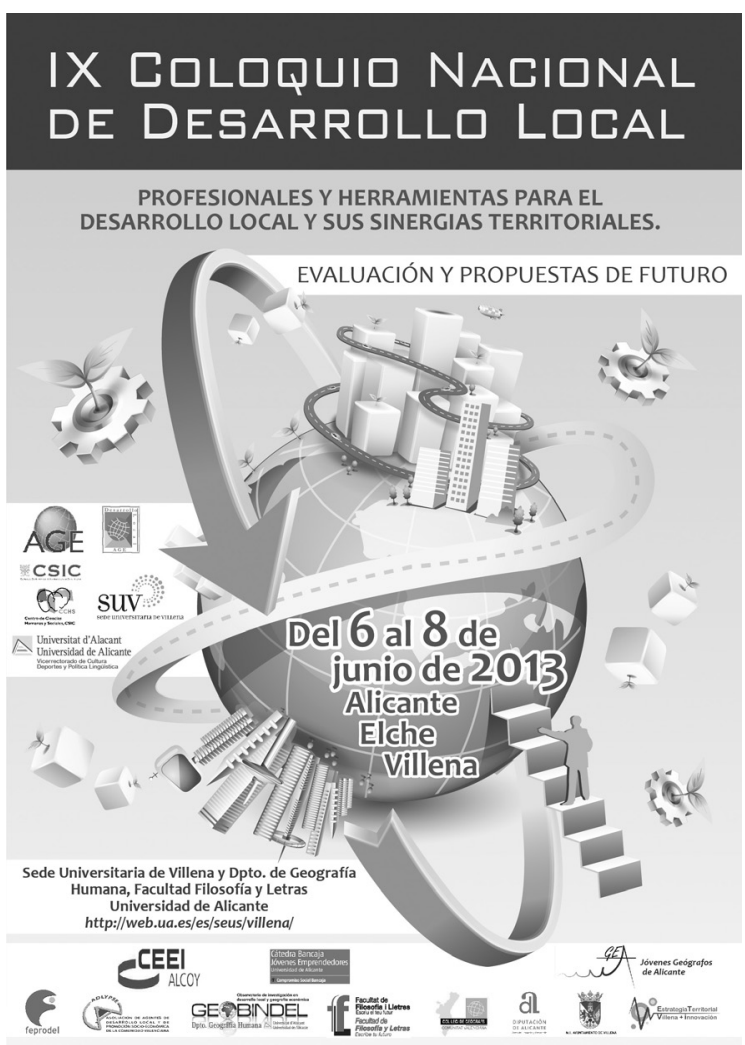


Este libro ha sido examinado y valorado por evaluadores ajenos a la Universidad de Alicante, con el fin de garantizar la calidad científica del mismo.

Publicacions de la Universitat d'Alacant

03690 Sant Vicent del Raspeig

Publicaciones@ua.es

http://publicaciones.ua.es

Telèfon: 965903480

(C) Antonio Martínez Puche, Xavier Amat Montesinos,

Isabel Sancho Carbonell y Daniel Sanchiz Castaño (eds.), 2016

(C) d'aquesta edició: Universitat d'Alacant

ISBN: 978-84-16724-00-0

Dipòsit legal: A 92-2016

Disseny de coberta: candela ink

Composició: Página Maestra (Miguel Ángel Sánchez Hernández)

Impressió i enquadernació: Guada Impresores

\section{unte \\ Unión de Editoriales
Universitarias Españolas \\ WWW.une.es
WWA}

Esta editorial es miembro de la UNE, cosa que garantiza la difusión y comercialización nacional y internacional de sus publicaciones.

Reservados todos los derechos. Cualquier forma de reproducción, distribución, comunicación pública o transformación de esta obra sólo puede ser realizada con la autorización de sus titulares, salvo excepción prevista por la ley. Diríjase a CEDRO (Centro Español de Derechos Repográficos, www.cedro.org) si necesita fotocopias o escanear algún fragmento de esta obra. 


\title{
AGRICULTURA, PAISAJE Y TURISMO COMO MOTOR DE DESARROLLO LOCAL: EL CASO DE LA ACTIVIDAD VITIVINÍCOLA EN LA COMARCA DEL PRIORAT (CATALUNYA)
}

\author{
Eloi Guinjoan Cesena \\ Universitat Autònoma de Barcelona, Departamento de Geografía \\ eloi.guinjoan@uab.cat
}

\section{RESUMEN}

Desde los años 80 hasta la actualidad, la comarca del Priorat (Tarragona) ha protagonizado un proceso de desarrollo local impulsado por la actividad vitivinícola, gracias a la adopción de un nuevo modelo productivo y al papel determinante que ha jugado la población local. En esta comunicación, se pretenden analizar los cambios de usos y cubiertas del suelo que ha propiciado dicho proceso. Los resultados más relevantes apuntan a una cierta recuperación de parcelas para el cultivo de la vid en las zonas más aptas, contrarrestando las dinámicas generales de abandono de tierras y transición forestal.

Palabras clave: vitivinicultura, paisaje, turismo, Priorat, cambios de usos/ cubiertas del suelo

AGRICULTURE, LANDSCAPE AND TOURISM AS LOCAL

DEVELOPMENT DRIVERS: THE CASE OF VINICULTURE IN THE

PRIORAT REGION (CATALONIA, NE SPAIN)

\section{Abstract}

Since the 80's to the present, the Priorat region (Catalonia) has undergone a process of local development driven by the viniculture, through the success of a new production model and the key role that local people have played. In this communication, we seek to analyze the land-use/land-cover changes this process has led to. The most significant changes relate to the recovery of several plots for growing vines in those areas with better characteristics for 
viticulture. This phenomenon has countered the overall land abandonment and forest transition dynamics.

Key words: viniculture, landscape, tourism, Priorat, land-use/land-cover change

\section{INTRODUCCIÓN}

Después de un siglo entero sumida en una grave crisis productiva, la comarca catalana del Priorat, un pequeño territorio de media montaña situado en el interior de la provincia de Tarragona, ha experimentado, desde finales de los años 80 hasta la actualidad, un importante renacimiento económico y demográfico. La clave de este proceso evolutivo ha sido la revalorización de la actividad vitivinícola, su vinculación con el territorio y la creación de sinergias con otros sectores productivos (MOLLEVÍ, 2007).

Paralelamente, ha surgido una nueva consciencia social, de modo que la población local ha comprendido que un paisaje cuidado y con personalidad es un complemento excelente para la actividad agraria, al mismo tiempo que fomenta el turismo (NOGUÉ, 2010). A su turno, ésta es la actividad más importante de un sector terciario que ya aglutina la mayor parte de ocupación en la comarca y, junto con la agricultura, sostiene la economía local (IDESCAT, 2013).

Dada la importancia que cobra el paisaje en el nuevo contexto socioeconómico del Priorat, resulta de gran relevancia prestar atención sobre los múltiples elementos que lo definen, motivo por el cual cobran especial protagonismo los usos y las cubiertas del suelo que encontramos en el territorio.

Así, en la presente comunicación, se pretenden identificar los principales cambios de usos y cubiertas del suelo que se han producido en el Priorat durante su período de desarrollo local. Este objeto de estudio ha sido abordado a partir de un análisis estadístico de los censos agrarios de 1989 y 1999, combinado con un análisis espacial, mediante técnicas SIG, del Mapa de Cubiertas del Suelo de Catalunya (MCSC) de 1993 y de 2005-07.

Entre los resultados obtenidos cabe destacar un notable incremento de la superficie dedicada al cultivo de la vid, que se ha producido a partir de la recuperación de parcelas abandonadas. Así, pese que a escala comarcal se han seguido manifestando los efectos de dinámicas generales como el abandono de cultivos y la transición forestal, a escala local podemos identificar determinadas zonas del Priorat donde ha habido un cierto cambio de tendencia, solamente explicable a partir del proceso de desarrollo local experimentado por la comarca

\section{UNA BREVE REFLEXIÓN TEÓRICA}

El desarrollo local surge como nuevo paradigma de desarrollo territorial a partir de los años 70, cuando algunos gobiernos locales y regionales deciden adaptar- 
se al nuevo orden económico mundial propiciando la activación de la economía y las sociedades locales a partir del aprovechamiento de los recursos endógenos (HERNANDO, 2007). Así pues, este modelo se basa en adaptar las ventajas competitivas locales al nuevo contexto global, y parte de la hipótesis según la cual todos los territorios tienen un conjunto de recursos propios de carácter económico, humano, cultural e institucional (VÁZQUEZ BARQUERO, 2000).

En el caso del Priorat, el recurso local que ha impulsado el desarrollo de la comarca ha sido la actividad vitivinícola, y su introducción en el mercado internacional se ha conseguido a través de un nuevo modelo de producción basado en la calidad, la singularidad de los vinos y su vinculación con el territorio (MOLLEVÍ, 2007; PAZ RICO, 2008).

A efectos territoriales, la transformación económica de las áreas rurales, como ha sucedido en el caso del Priorat, conlleva inevitablemente la aparición de unas dinámicas opuestas de extensificación e intensificación del uso del suelo, que a su turno se traducen en cambios en las cubiertas del suelo (MARTÍNEZ ILLA et al., 1988). Entendemos por "uso del suelo" las formas de explotación que hace de éste el ser humano, mientras que la "cubierta del suelo" se limita a su condición biofísica (MEYER y TURNER, 1996). En todo caso, ambos conceptos constituyen un componente esencial del paisaje (SERRA y PINTÓ, 2005; BADIA et al., 2008; ESTANY et al., 2010), que a su turno se trata de uno de los elementos clave del proceso de desarrollo local experimentado en la comarca del Priorat (MOLLEVÍ, 2007).

Asimismo, cuando tratamos con paisajes tradicionales, el papel de los usos y las cubiertas del suelo resulta excepcionalmente relevante, debido al hecho que son paisajes fruto de las actividades productivas primarias que la población local ha desarrollado al largo de los siglos, y cualquier cambio repentino de usos del suelo a grande escala puede tener enormes consecuencias (AGNOLETTI, 2007). En el caso del Priorat, además, esta amenaza aparece multiplicada, ya que aparte de tratarse de un paisaje tradicional -y más concretamente, cultural y agrario- modelado lentamente desde la Edad Media hasta la actualidad, el modelo socioeconómico actual de la comarca depende en gran medida de la calidad de su paisaje, en tanto que complemento de la actividad agraria y elemento generador de turismo (NOGUÉ, 2010).

\section{Presentación del ÁRea de estudio}

La comarca del Priorat está situada en el sur de Catalunya, en el sector interior de la provincia de Tarragona, y tiene una superficie de $499 \mathrm{~km}^{2}$. Actualmente integrada por 23 municipios, la comarca ha sido tradicionalmente dividida en cuatro zonas en base a criterios relacionados con la topografía, el suelo y las actividades agrarias (MARGALEF y TASIAS, 1985) (figura 1). 


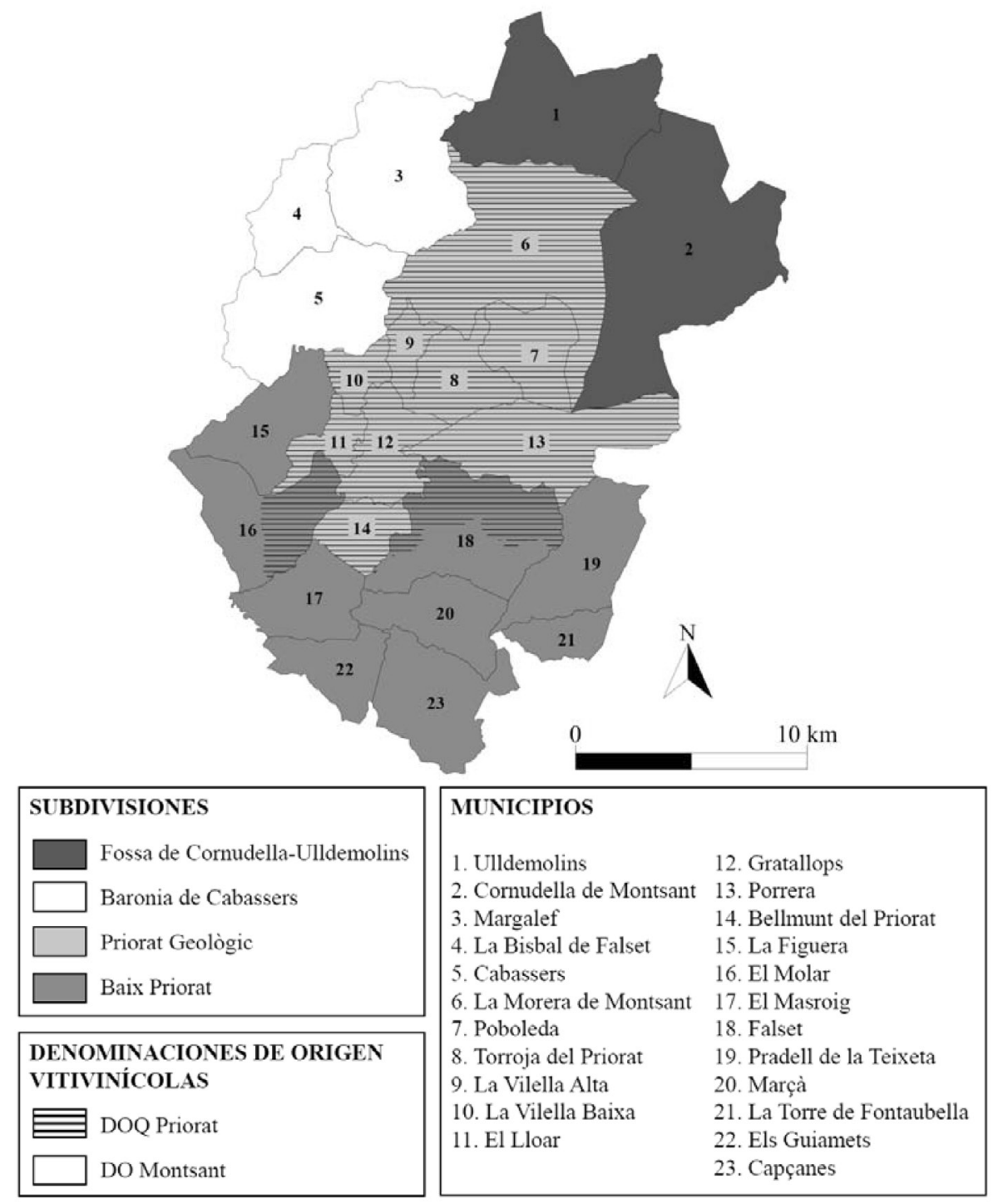

Figura 1. Mapa del área de estudio.

Fuente: elaboración propia.

A partir de las grandes descripciones de IGLÉSIAS (1974) y ANGUERA (1984), así como de los datos actuales sobre cultivos georreferenciados en el MCSC 2005-07, podemos diferenciar rápidamente estas cuatro zonas, una tarea necesaria para comprender mejor los resultados del análisis de cambios de usos y cubiertas del suelo que presentaremos más adelante. 
En primer lugar, hay que destacar la zona del Priorat Geològic, correspondiente al sector central de la comarca. Esta zona se caracteriza por un relieve ondulado, con fuertes pendientes, y un suelo formado por pizarras primarias, popularmente conocidas como "licorellas". Pese a los bajos rendimientos agrícolas que se obtienen sobre este tipo de suelos, las licorellas permiten la elaboración de unos vinos de elevadísima calidad, y son el carácter diferencial de la Denominación de Origen Calificada (DOC) Priorat, que prácticamente coincide con los límites del Priorat Geològic. Como corresponde, pues, se trata de una zona con un marcado monocultivo vitícola.

Por otro lado, tenemos la zona del Baix Priorat, situada al sur de la comarca, con un relieve más llano y propicio para los asentamientos y para la mecanización de la agricultura, especialmente en su sector más occidental. En este ámbito, es muy importante el cultivo de la vid y los avellanos, así como también los olivos, aunque en menor proporción.

En tercer lugar, hay que hablar de la Fossa de Cornudella-Ulldemolins, correspondiente al valle situado entre la imponente Serra del Montsant y las Muntanyes de Prades. En este caso, cobra especial importancia el cultivo del almendro.

Finalmente, tenemos la Baronia de Cabassers, una zona ubicada al extremo noroeste de la comarca, con un relieve montañoso, y muy próxima a la comarca contigua de las Garrigues, de larga tradición oleícola. Así pues, esta zona se caracteriza esencialmente por el cultivo del olivo.

Pese al menor protagonismo de la vid en estas tres últimas zonas de la comarca, todas ellas se inscriben dentro del ámbito de la DO Montsant, considerada como una de las denominaciones de origen vitivinícolas de Catalunya con más potencial a corto plazo (PAZ RICO, 2008).

\section{El pRoceso de DESARROLLO LOCAL}

\subsection{Contexto previo: un siglo de crisis de producción}

Hasta los años 80, la comarca del Priorat estaba inmersa en un contexto económico caracterizado por una elevada polarización agraria y una especialización persistente en este sector, estructurado en explotaciones familiares generalmente poco rentables (FLOS BASSOLS, 1984). Esta baja productividad histórica es debida tanto a factores climáticos -períodos de sequía en verano y heladas tardías en invierno-, como a factores geológicos -baja calidad de los suelos y topografía accidentada- (MARGALEF y TASIAS, 1985).

A todos estos factores hay que sumarles la crisis de la filoxera, una plaga mortífera que llegó a la comarca el año 1893 y que arrasó todas las vides del Priorat. Pese a los primeros esfuerzos de recuperación, la comarca quedó sumida en una grave crisis de producción, que se manifestó en términos agrícolas, comerciales, técnicos, económicos y demográficos. Así, la filoxera fue el ele- 
mento detonante de la crisis, pero lo que sucedió en realidad durante el siglo $\mathrm{XX}$ es que la comarca estaba pagando su excesiva acomodación económica durante la previa etapa de esplendor (PERPINYÀ GRAU, 1932).

\subsection{Origen y filosofía del proceso: un acto de fe en la tierra}

Esta crisis de producción se acabó alargando hasta los años 80 del siglo XX, cuando varios enólogos, algunos de ellos foráneos, conscientes del enorme potencial de la licorella, se aventuraron a comprar tierras en la zona del Priorat Geològic, plantaron nuevas variedades de vid y apostaron por nuevos sistemas de elaboración y comercialización del vino (SABATÉ, 2001).

Siguiendo los principios teóricos del desarrollo local, estos enólogos pioneros se preocuparon, desde el sector vitivinícola, por adaptar las condiciones locales del Priorat al mercado internacional. Así, conscientes de la dificultad de vender vinos de alta graduación alcohólica en garrafas, apostaron por la introducción de nuevas variedades de vid -con el objetivo de rebajar esta gradación alcohólica- y por la venta embotellada del vino resultante. Obviamente estos cambios requirieron importantes inversiones, pero tuvieron su efecto, ya que finalmente el Priorat consiguió aquello que todavía no había logrado hasta el momento: introducir sus vinos al mercado extranjero con éxito y a precios razonables (MOLLEVÍ, 2007).

Este nuevo modelo de producción, aparte de estar basado en la calidad, se caracteriza por un elevado grado de vinculación con la tierra. Así, los enólogos -y por extensión, los viticultores que decidieron invertir y montar también su propia bodega- procuran elaborar vinos con personalidad propia y difícilmente imitables, aprovechando las características físicas singulares del territorio (PAZ RICO, 2008). Sin embargo, las nuevas y las antiguas bodegas conviven aún con las cooperativas, que han sabido adaptarse al nuevo modelo de producción, basado en la calidad, y permiten la supervivencia del pequeño viticultor en medio de empresas enológicas con grandes capitales.

En todo caso, resulta significativo que aquellos rasgos que siempre se habían considerado obstáculos para una actividad agraria rentable -básicamente el suelo de licorella y el clima, que limitan seriamente los niveles de productividad- con el nuevo modelo de producción pasaron a ser elementos determinantes para la calidad del vino y para su diferenciación dentro de un mercado global que cada vez aprecia más la singularidad y el valor local de los productos agroalimentarios (MOLLEVÍ, 2007).

Así pues, ha surgido una nueva filosofía dentro del sector vitivinícola de la comarca. En palabras de uno de los enólogos pioneros que llegó al Priorat, el francés René Barbier, ahora cada botella de vino "es una historia, es la personalidad potente y única del Priorat" (DOMÈNECH et al., 1999). Esta creciente vinculación entre el vino y la tierra ha generado en la comarca una serie de sinergias entre la agricultura, el territorio y el paisaje (MOLLEVÍ, 2007). 
Asimismo, los agricultores han comprendido que el paisaje es un complemento excelente para la actividad agraria, al mismo tiempo que fomenta el turismo (NOGUÉ, 2010), una actividad que actualmente encabeza un sector terciario con un peso creciente en el cómputo comarcal, pero que depende extremadamente de la salud del sector primario, pues el atractivo paisajístico del Priorat subyace en su carácter agrario y cultural.

Como resultado del renacimiento vitivinícola, han surgido nuevos conceptos con el fin de poder relacionar el vino elaborado con su origen. En este sentido, se considera que el vino que actualmente se elabora en el Priorat es, utilizando un término francés, vin de terroir, traduciendo terroir por "tierra", "terreno" o "territorio". Asimismo, han aparecido varias figuras de catalogación de vinos, como el vi de clos -vino elaborado con uvas de una única finca- o el vino de autor -vino que ha contado con el control de un enólogo en todas sus fases de elaboración-, figuras que no hacen más que añadir prestigio y reconocimiento a estos productos agroalimentarios de alta calidad.

\subsection{Agentes implicados}

Pese al imprescindible acto de fe que hicieron los enólogos pioneros que arriesgaron capital en una comarca sosegada por la crisis, no hay que olvidar que el gran mérito de la revalorización de los vinos del Priorat corresponde a la población local. En efecto, los prioratinos son los únicos responsables de haber convertido su comarca en sinónimo de vino a lo largo de varias generaciones, de haber mantenido viva la tradición durante la crisis del siglo XX y de haber optado por unas variedades de vid y unas técnicas de cultivo determinadas. Asimismo, la implicación local y el despertar de un nuevo sentimiento de identidad dentro de la comarca, propició que la introducción de nuevas variedades de vid -básicamente cabernet sauvignon, merlot y syrah- se combinara con la recuperación de la garnacha, una variedad que se había cultivado en el Priorat desde el siglo XIV (PAZ RICO, 2008).

No obstante, el proceso de desarrollo local del Priorat ha traído a la comarca personas con un perfil muy distinto. Así, actualmente, encontramos una gran diversidad de agentes dentro del sector vitivinícola comarcal: las antiguas bodegas, las cooperativas, los viticultores locales que se han adaptado al nuevo modelo de producción, los enólogos pioneros y sus discípulos, los recién llegados atraídos por el glamour generado, grandes empresas enológicas y incluso algunos grupos inversores con finalidades puramente especulativas, además de entidades como los consejos reguladores de las denominaciones de origen, la escuela de enología de Falset o el parque tecnológico del vino, el "Vitec".

En referencia a les denominaciones de origen vitivinícolas, cabe destacar que, como ya fue comentado, el proceso de innovación de la actividad vitivinícola surgió en la zona pizarrosa del Priorat Geològic, debajo el paraguas de la DO Priorat, que fue reconocida como DOC a partir del año 2000. Sin 
embargo, el nuevo modelo de producción rápidamente se extendió por el resto de la comarca, hasta el punto que actualmente, la DO Montsant -que hasta el año 2001 era una simple subzona de la DO Tarragona-, se considera una denominación de origen con una gran proyección a corto plazo, favorecida por un territorio con un relieve más propicio para la mecanización y con unas vides más jóvenes.

5. ANÁlisis de los CAMbios de usos y CUBIERTAS Del SUELO PROPICIADOS POR EL DESARROLLO LOCAL

\subsection{Evolución de la superficie cultivada}

Pese a la revalorización de la actividad agrícola y de los productos agroalimentarios locales que ha conllevado el proceso de desarrollo local del Priorat, durante este período se ha seguido produciendo una disminución de la superficie cultivada en la comarca. Así, según datos del censo agrario de 1989 y del MCSC 2005-07, entre 1989 y 2007 se ha perdido un 10,5\% de las tierras cultivadas del Priorat. No obstante, esta evolución no se presenta de forma uniforme por todo el territorio, a la vez que esconde significativas diferencias según los principales cultivos de la comarca: la vid, el olivo y la fruta seca.

Para empezar, hay que hablar de la vid, el cultivo por excelencia del Priorat a lo largo de la historia, y el que determina en mayor grado su identidad. Como motor de desarrollo que ha sido, no es de extrañar que, más de un siglo después de la llegada de la filoxera en la comarca, finalmente la superficie cultivada con vid haya vuelto a crecer a partir de los años 80 . Este incremento ha sido bastante generalizado, pues se ha percibido en 17 de los 23 municipios de la comarca, pero ha destacado especialmente en la zona pizarrosa del Priorat Geològic (cuadro 1), el ámbito que ofrece mayores posibilidades dentro del nuevo modelo productivo basado en la calidad, la singularidad y la vinculación con el terroir.

Cuadro 1. Evolución de la superficie de cultivo de vid (1989-2007)

\begin{tabular}{|l|c|c|c|c|c|c|}
\hline \multirow{2}{*}{\multicolumn{1}{|c|}{ Zona }} & \multicolumn{3}{c|}{ Superficie (ha) } & \multicolumn{3}{c|}{ Crecimiento (\%) } \\
\cline { 2 - 7 } & $\mathbf{1 9 8 9}$ & $\mathbf{1 9 9 9}$ & $\mathbf{2 0 0 7}$ & $\begin{array}{c}\mathbf{1 9 8 9 -} \\
\mathbf{1 9 9 9}\end{array}$ & $\begin{array}{c}\mathbf{1 9 9 9 -} \\
\mathbf{2 0 0 7}\end{array}$ & $\begin{array}{c}\mathbf{1 9 8 9} \\
\mathbf{2 0 0 7}\end{array}$ \\
\hline Priorat Geòlogic & 803 & 1003 & 1753 & 24,9 & 74,8 & 118,3 \\
\hline Baix Priorat & 1587 & 1714 & 1941 & 8,0 & 13,3 & 22,3 \\
\hline $\begin{array}{l}\text { Fossa de Cornudella- } \\
\text { Ulldemolins }\end{array}$ & 166 & 148 & 189 & $-10,8$ & 27,6 & 13,7 \\
\hline Baronia de Cabassers & 57 & 47 & 71 & $-17,5$ & 51,9 & 25,3 \\
\hline TOTAL PRIORAT & 2613 & 2912 & 3955 & 11,4 & 35,8 & 51,4 \\
\hline
\end{tabular}

Fuente: elaboración propia a partir de los censos agrarios de 1989 y 1999 y del MCSC 2005-07. 
En cambio, en el resto de zonas de la comarca, la recuperación de la vid no fue tan rápida, de modo que se siguieron abandonando parcelas con cultivo vitícola hasta finales de los años 90. Así, no fue hasta principios de siglo XXI que la superficie cultivada con vid empezó a aumentar de forma generalizada fuera del Priorat Geològic, precisamente coincidiendo con la constitución de la DO Montsant. Por otro lado, entre 1989 y 2007 el cultivo vitícola solo disminuyó en algunos municipios de la franja oeste de la comarca, donde la tradición oleícola siempre ha sido mayor, y donde, como veremos a continuación, este otro cultivo también se ha visto consolidado durante el periodo de desarrollo local que ha experimentado la comarca del Priorat.

Resulta el momento, pues, de hablar del cultivo del olivo. Según las estadísticas históricas aportadas por MARGALEF y TASIAS (1985), podemos deducir que en la mayor parte de la comarca, este cultivo adquirió importancia solo a partir del siglo XX, como substituto de la vid. La excepción la marca, en este caso, la zona de la Baronia de Cabassers, donde a causa de sus características geomorfológicas y su proximidad a la comarca oleícola de las Garrigues, el cultivo del olivo siempre ha sido predominante.

En todo caso, la evolución de la superficie de olivos en la comarca no ha sido constante durante el periodo de desarrollo local, y podemos identificar dos etapas muy diferenciadas: una primera de incremento de superficie, y una segunda de pérdida, coincidiendo con la consolidación del cultivo vitícola (cuadro 2).

Cuadro 2. Evolución de la superficie de cultivo de olivo (1989-2007)

\begin{tabular}{|l|c|c|c|c|c|c|}
\hline \multirow{2}{*}{\multicolumn{1}{|c|}{ Zona }} & \multicolumn{3}{c|}{ Superficie (ha) } & \multicolumn{3}{c|}{ Crecimiento (\%) } \\
\cline { 2 - 8 } & $\mathbf{1 9 8 9}$ & $\mathbf{1 9 9 9}$ & $\mathbf{2 0 0 7}$ & $\begin{array}{c}\mathbf{1 9 8 9} \\
\mathbf{1 9 9 9}\end{array}$ & $\begin{array}{c}\mathbf{1 9 9 9 -} \\
\mathbf{2 0 0 7}\end{array}$ & $\begin{array}{c}\mathbf{1 9 8 9} \\
\mathbf{2 0 0 7}\end{array}$ \\
\hline Priorat Geòlogic & 340 & 517 & 360 & 52,1 & $-30,4$ & 5,8 \\
\hline Baix Priorat & 634 & 1136 & 803 & 79,2 & $-29,3$ & 26,7 \\
\hline $\begin{array}{l}\text { Fossa de Cornudella- } \\
\text { Ulldemolins }\end{array}$ & 312 & 393 & 240 & 26,0 & $-38,9$ & $-23,1$ \\
\hline Baronia de Cabassers & 984 & 1119 & 1210 & 13,7 & 8,1 & 23,0 \\
\hline TOTAL PRIORAT & 2270 & 3164 & 2613 & 39,4 & $-17,4$ & 15,1 \\
\hline
\end{tabular}

Fuente: elaboración propia a partir de los censos agrarios de 1989 y 1999 y del MCSC 2005-07.

Efectivamente, podemos ver cómo durante la década de los 90, la superficie dedicada al cultivo del olivo creció en todas las zonas de la comarca -y prácticamente en todos los municipios-, como ya venía haciendo desde años anteriores. Sin embargo, a partir de los años 2000, una vez la "revolución del vino" se consolidó en la comarca, el cultivo del olivo volvió a disminuir otra 
vez -excepto en la Baronia de Cabassers-, confirmando que en la mayor parte de los casos, se trataba de un cultivo substitutorio de la vid, a la espera de la revalorización de los vinos del Priorat.

Pese a estas dos etapas tan diferenciadas y al cómputo global que nos indica un crecimiento del $15,1 \%$ de la superficie de olivos entre 1989 y 2007, la dinámica decreciente de los últimos años nos sugiere que el incremento de superficie de los años 90 se produjo únicamente debido a la inercia de una crisis vitivinícola que justo empezaba a ver su fin. Por el contrario, la consolidación del modelo de desarrollo local adoptado en el Priorat, basado en la actividad vitivinícola, se ha acabado traduciendo en un retroceso del cultivo del olivo, como hemos visto a partir de los años 2000 en prácticamente toda la comarca, exceptuando la zona ya tradicionalmente oleícola de la Baronia de Cabassers.

Finalmente, cuanto a los cultivos de fruta seca, hay que precisar que en la comarca del Priorat se limitan a almendros y avellanos. Después de adquirir importancia durante el siglo XX a raíz de la crisis vitivinícola, estos dos cultivos llegaron a ser los más importantes de la comarca durante los años 80 (MARGALEF y TASIAS, 1985). Sin embargo, la entrada de España en la Comunidad Económica Europea sumió el sector de la fruta seca en una grave crisis que también se notaría en el Priorat, donde la superficie cultivada con almendros y avellanos disminuyó de 6.571 hectáreas a tan solo 3.734 entre los años 1989 y 1999 (cuadro 3).

Cuadro 3. Evolución de la superficie de cultivo de leñosos de fruta seca (1989-2007)

\begin{tabular}{|l|c|c|c|c|c|c|}
\hline \multirow{2}{*}{\multicolumn{1}{|c|}{ Zona }} & \multicolumn{3}{c|}{ Superficie (ha) } & \multicolumn{3}{c|}{ Crecimiento (\%) } \\
\cline { 2 - 8 } & $\mathbf{1 9 8 9}$ & $\mathbf{1 9 9 9}$ & $\mathbf{2 0 0 7}$ & $\begin{array}{c}\mathbf{1 9 8 9 -} \\
\mathbf{1 9 9 9}\end{array}$ & $\begin{array}{c}\mathbf{1 9 9 9 -} \\
\mathbf{2 0 0 7}\end{array}$ & $\begin{array}{c}\mathbf{1 9 8 9} \\
\mathbf{2 0 0 7}\end{array}$ \\
\hline Priorat Geòlogic & 1441 & 824 & 577 & $-42,8$ & $-30,0$ & $-60,0$ \\
\hline Baix Priorat & 2918 & 1785 & 1784 & $-38,8$ & 0,0 & $-38,9$ \\
\hline $\begin{array}{l}\text { Fossa de Cornudella- } \\
\text { Ulldemolins }\end{array}$ & 1536 & 852 & 998 & $-44,5$ & 17,1 & $-35,0$ \\
\hline Baronia de Cabassers & 676 & 275 & 175 & $-59,3$ & $-36,5$ & $-74,2$ \\
\hline TOTAL PRIORAT & 6571 & 3734 & 3534 & $-43,2$ & $-5,4$ & $-46,2$ \\
\hline
\end{tabular}

Fuente: elaboración propia a partir de los censos agrarios de 1989 y 1999 y del MCSC 2005-07.

Sin embargo, como hemos comentado, este fue un factor totalmente ajeno al proceso de desarrollo local del Priorat, ya que responde a una crisis de escala estatal. Por otro lado, los verdaderos efectos de dicho proceso sobre el cultivo de almendros y avellanos se manifestaron a partir de los años 2000, cuando se 
empezaron a percibir dinámicas muy diferentes en cada zona de la comarca, en función de la importancia de la vid y del olivo.

Así, por un lado, la superficie dedicada a los cultivos de fruta seca siguió disminuyendo de forma irremediable en la Baronia de Cabassers -cada vez más especializada en el cultivo del olivo- y en el Priorat Geològic -donde el renacimiento vitivinícola cobró mayor protagonismo-. Por otro lado, en el resto del territorio comarcal, donde no se ha producido una especialización tan marcada en ninguno de los dos cultivos anteriores, la superficie de cultivo de almendros y avellanos no ha sufrido grandes variaciones y incluso ha tendido a crecer pese a la fuerte crisis que sigue golpeando el sector de la fruta seca, un hecho que demuestra que el nuevo auge vitivinícola del Priorat ha conllevado una revalorización de toda la actividad agraria en general.

\subsection{Evolución de las cubiertas de vegetación espontánea}

Mientras que la superficie cultivada ha disminuido durante el proceso de desarrollo local de la comarca, la superficie boscosa ha crecido substancialmente y la de matorral se ha mantenido relativamente estable.

Así, según datos de la primera y la tercera edición del MCSC, entre 1993 y 2007 la superficie cubierta por bosque ha aumentado un 16,0\% en toda la comarca, un hecho que no deja de ser resultado de una dinámica propia de toda la montaña mediterránea, como es el abandono de cultivos, y que como acabamos de ver, el desarrollo local del Priorat no consiguió invertir, aunque si minimizar.

Este incremento boscoso ha sido especialmente importante en los sectores más montañosos del Priorat, así como también en aquellos municipios que, después de sufrir un abandono importante de tierras cultivadas durante el siglo $\mathrm{XX}$, no han sido capaces de recuperar la actividad agrícola debido a la menor adaptación de su terreno para el cultivo de la vid, como es el caso del extremo oriental del Baix Priorat.

Por el contrario, en las zonas donde la agricultura ofrece más posibilidades actualmente -el Priorat Geològic, gracias a la licorella, y el sector occidental del Baix Priorat, debido a su relieve más llano-, el menor abandono de tierras ha propiciado que la expansión del bosque también haya adquirido menos importancia.

En cuanto al matorral, su aparente estabilidad en términos de superficie esconde en realidad grandes variaciones locales. Así, entre 1993 y 2007, según datos de los MCSC, la superficie de matorral ha crecido notablemente en la corona norte de la comarca, correspondiente a la Serra del Montsant. Sin embargo, lo que resulta más significativo es que haya disminuido en toda la zona del Priorat Geològic, precisamente allí donde se ha producido una mayor recuperación de tierras para el cultivo vitícola.

En una línea similar, y a partir de la superposición del MCSC 1993 y 200507, podemos observar que los municipios donde ha habido un mayor porcentaje 
de superficie cubierta por vegetación espontánea -bosque, matorral o herbazalque entre 1993 y 2007 se ha convertido en área de cultivos, corresponden a las áreas con mejores características para el cultivo de la vid, es decir, el Priorat Geològic y el sector más occidental del Baix Priorat (figura 2).

En este sentido, cabe destacar que en los municipios de la Vilella Alta, Torroja del Priorat y Gratallops -todos pertenecientes al Priorat Geològic-, más del 10\% de las áreas que en 1993 estaban cubiertas por bosque, matorral o herbazal, en 2007 ya habían sido recuperadas para el desarrollo de la actividad agrícola, mayormente para el cultivo de la vid.

Así pues, podemos aceptar que el proceso de desarrollo local experimentado en el Priorat no ha impedido que se manifestaran los efectos de unas dinámicas comunes que vienen reproduciéndose las últimas décadas en toda la montaña mediterránea, como es el abandono de tierras cultivadas y la expansión de la superficie forestal. No obstante, este proceso de desarrollo local sí que ha atenuado claramente estas dinámicas, especialmente gracias al notable

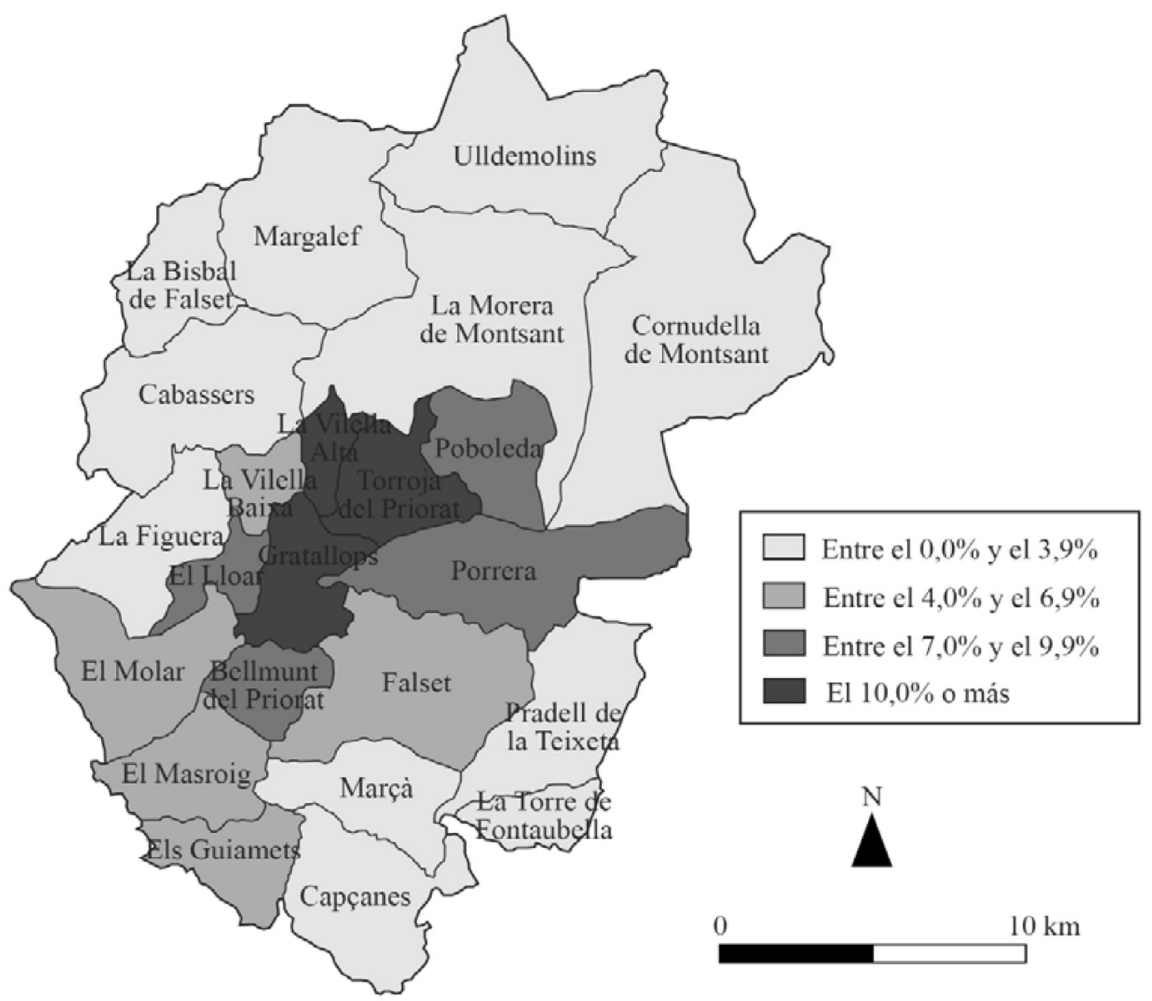

Figura 2. Mapa del porcentaje de vegetación espontánea transformada en cultivos entre 1993 y 2007 por municipios.

Fuente: elaboración propia a partir del MCSC 1993 y 2005-07. 
incremento de la superficie dedicada al cultivo de la vid, que ha propiciado una cierta recuperación de parcelas agrarias abandonadas en el Priorat Geològic -donde el suelo de licorella permite la elaboración de vinos de elevada calidad y marcada singularidad- y en el sector occidental del Baix Priorat -donde encontramos un relieve relativamente llano y propicio para la mecanización de la agricultura-.

\section{Conclusiones}

Desde los años 80 hasta la actualidad, la comarca del Priorat ha experimentado un proceso de desarrollo local impulsado por la actividad vitivinícola, y más concretamente, por una serie de mejoras que han permitido el desarrollo de un nuevo modelo productivo basado en la calidad de los vinos, su singularidad y un alto grado de vinculación con la tierra.

Pese al destacado papel que jugaron los enólogos pioneros que propiciaron estas mejoras, y pese a la diversidad de agentes que se han acabado involucrando en el sector vitivinícola de la comarca, el último responsable del proceso de desarrollo local del Priorat no deja de ser su población local, tanto por el papel que jugó durante el siglo de crisis, como también por haber comprendido la importancia de su paisaje y haberlo sabido integrar en la oferta agroalimentaria y turística, fortaleciendo asimismo el sentimiento de identidad local.

Cuanto a los resultados del proceso de desarrollo local, la producción de vinos de calidad dentro de la DOC Priorat y dentro de la DO Montsant, ambas con elevados porcentajes de exportación, demuestra que la vitivinicultura es una actividad generadora de valor añadido en la comarca, tanto de una forma directa -a través del producto-, como de forma indirecta -generando turismo-.

En referencia a las consecuencias territoriales de dicho desarrollo, hay que destacar que, si bien no ha conseguido invertir determinadas dinámicas generales del monte mediterráneo, como es el abandono de cultivos y la transición forestal, sí ha conseguido minimizarlas, especialmente a través de la recuperación de parcelas abandonadas que han sido aprovechadas de nuevo para el cultivo de la vid. Este fenómeno ha sido especialmente notorio en las zonas del Priorat Geològic -donde el medio físico ofrece mayores posibilidades para dotar los vinos de calidad y singularidad- y en el sector occidental del Baix Priorat -donde encontramos un relieve más adecuado para la actividad agrícola-.

Asimismo, aunque el nuevo auge de la vid ha acabado provocando, ya a partir de los años 2000, una cierta pérdida de cultivos de olivo, la creciente especialización de la zona de la Baronia de Cabassers en este cultivo, así como la parcial recuperación del almendro y el avellano en determinados sectores de la comarca -pese a la fuerte crisis que atraviesa el sector de la fruta seca- son claros síntomas de una revalorización de la actividad agraria en general, un fenómeno que, en el caso del Priorat, ha tenido como principales protagonistas a los pequeños agricultores locales. 
BIBLIOGRAFÍA

AGNOLETTI, M. (2007): "The degradation of traditional landscape in a mountain area of Tuscany during the 19th and 20th centuries: Implications for biodiversity and sustainable management", Forest Ecology and Management, $\mathrm{n}^{\mathrm{o}} 249$, p. 5-17.

ANGUERA, P. (1984): "El Priorat", en ENCICLOPÈDIA CATALANA (ed.) Gran Geografia Comarcal de Catalunya, vol. 11, Barcelona, Fundació Enciclopèdia Catalana.

BADIA, A.; OTERO, I.; MANEJA, R.; ESTANY, G. y BOADA, M. (2008): "Canvi global i paisatge a la Costa del Tet-Mont-rodon (Matadepera, Vallès Occidental). Analitzar el passat per planificar el futur (1956-2006)", Documents d'Anàlisi Geogràfica, no 52, p. 31-48.

DOMĖNECH, J.; PORTA, C. y POU, F. (1999): "La força del vi” [vídeo documental], 30 Minuts, Televisió de Catalunya, 28 de febrero de 1999.

ESTANY, G.; BADIA, A.; OTERO, I. y BOADA, M. (2010): “The socioecological transformation from rural to residential landscapes in mediterranean metropolis. Maps and vanishing voices from the municipality of Matadepera (Barcelona Metropolitan Region)", Global Environment, n 5, p. 8-38.

FLOS BASSOLS, A. (1984): "La localització econòmica de Catalunya", en BANCO DE BILBAO (ed.) L'economia a Catalunya d'avui i demà, Barcelona, Banco de Bilbao.

HERNANDO, M. (2007): El desenvolupament local, Barcelona, Editorial UOC. IDESCAT, www.idescat.cat [consulta: febrero-abril 2013].

IGLÉSIAS, J. (1974): "Priorat", en SOLÉ SABARÍS, L. (dir.) Geografia de Catalunya, vol. III, Barcelona, Aedos, p. 141-168.

MARGAlEF, J. y TASIAS, J. (1985): El Priorat. Anàlisi d'una crisi productiva, Barcelona, Caixa d'Estalvis de Catalunya.

MARTÍNEZ ILLA, S.; NUNES, J. y VALERO, J. C. (1988): "Evolució dels usos del sol. Inventari i anàlisi”, Documents d'Anàlisi Geogràfica, nº12, p. 39-57.

MEYER, W. B. y TURNER, B. L. (1996): "Land-use/land-cover change: challenges for geographers", GeoJournal, no 39, p. 237-240.

MOLLEVÍ, G. (2007): La geografía de la vid y el vino en Cataluña, Barcelona, DaVinci.

NOGUÉ, J. (2010): Paisatge, territori i societat civil, València, Edicions 3 i 4.

PAZ RICO, A. (2008): El secret del vi del Priorat: el terrer, la història o la seva gent?, Valls, Cossetània Edicions.

PERPINYÀ GRAU, R. (1932): La crisi del Priorat. L'interès col-lectiu econòmic a Catalunya $i$ València, Reus, Centre de Lectura, 1982.

SABATÉ, J. (2001): "Desenvolupament socioeconòmic dels pobles de l'antic Priorat", en Revista del Centre de Lectura de Reus, n 68, p. 4-7. 
SERRA, I. y PINTÓ, J. (2005): "La transformació del paisatge del delta de la Tordera en els darrers cent cinquanta anys. Una anàlisi per mitjà dels canvis en els usos i les cobertes del sòl", Documents d'Anàlisi Geogràfica, $\mathrm{n}^{\circ}$ 46, p. 81-102.

VÁZQUEZ BARQUERO, A. (2000): "Desarrollo local y territorio", en PÉREZ RAMÍREZ, B. y CARRILLO, E. (coord.) Desarrollo local: manual de uso, Madrid, Esic Editorial, p. 93-107. 